\author{
Nota científica \\ (Short communication)
}

\title{
OBSERVACIONES DEL RASCÓN PINTO, PARDIRALLUS MACULATUS (AVES: RALLIDAE) EN EL CENTRO-OCCIDENTE DE VERACRUZ
}

\author{
OBSERVATIONS OF THE SPOTTED RAIL, PARDIRALLUS MACULATUS (AVES: \\ RALLIDAE) IN CENTRAL-WESTERN VERACRUZ
}

\author{
AXEL FUENTES MORENO ${ }^{1 *}$, MACARIO FERNÁNDEZ POPO², VÍCTOR VÁSQUEZ CRUZ², ABIGAIL \\ MORA REYES ${ }^{2}$, OSCAR CID MORA ${ }^{2}$ \\ ${ }^{1}$ Colegio de Postgraduados Campus Montecillo. Carretera México-Texcoco Km. 36.5, Montecillo, Texcoco. Estado de México. \\ C.P. 56230.<euphagus@gmail.com> \\ ${ }^{2}$ Facultad de Ciencias Biológicas y Agropecuarias, Universidad Veracruzana. Camino Peñuela -Amatlán S/N, Peñuela Mpio. de \\ Amatlán de los Reyes, Veracruz. C.P. 94952. <biol.fernandez92@gmail.com>; <victorbiolvc@ gmail.com>; \\ <more.xenarthra@gmail.com>; <racso10390@gmail.com> \\ *Autor de correspondencia: <euphagus@gmail.com> \\ Recibido: 14/03/2018; aceptado: 11/06/2019; publicado en línea: 10/07/2019 \\ Editor responsable: Ricardo Rodríguez Estrella
}

Fuentes-Moreno, A., Fernández-Popo, M., Vásquez-Cruz, V., Mora-Reyes, A., Cid-Mora, O. (2019) Observaciones del rascón pinto, Pardirallus maculatus (Aves: Rallidae) en el centro-occidente de Veracruz. Acta Zoológica Mexicana (nueva serie), 35, 1-5. https://doi.org/10.21829/azm.2019.3502223

RESUMEN. Presentamos los primeros registros del rascón pinto (Pardirallus maculatus) en el centro-oeste de Veracruz, su presencia en esta área puede explicarse por la gran capacidad de dispersión o vagabundeo de la especie o bien, por sesgos en el conocimiento de su distribución. Asimismo, reportamos el consumo de lenteja de agua (Lemnaceae) para este rascón.

Palabras clave: Aves acuáticas; lentejas de agua; tular; picoteo; Amatlán de los Reyes; Orizaba; Altas Montañas de Veracruz

Fuentes-Moreno, A., Fernández-Popo, M., Vásquez-Cruz, V., Mora-Reyes, A., Cid-Mora, O. (2019) Observations of the spotted rail, Pardirallus maculatus (Aves: Rallidae) in central-western Veracruz. Acta Zoológica Mexicana (nueva serie), 35, 1-5. https://doi.org/10.21829/azm.2019.3502223

ABSTRACT. We report the first records of the Spotted Rail (Pardirallus maculatus) in central-western Veracruz. The presence of Spotted rails in this area can be explained by the high dispersal capabilities or wandering behavior of the species but also it could be due to the fact that there are biases in the knowledge of its distribution. Also, we report the consumption of duckweed plants (Lemnaceae) for this rail.

Key words: Waterbirds; duckweeds; cattail marsh; pecking; Amatlán de los Reyes; Orizaba; Altas Montañas de Veracruz 
La familia Rallidae posee una distribución casi cosmopolita, sin embargo, varias especies del grupo viven en hábitats poco accesibles o presentan conductas crípticas, por lo que el conocimiento de la biología, ecología y tamaño poblacional de muchas de ellas es bastante limitado (Taylor \& Van Perlo, 1988; Howell \& Webb, 1995; Del Barco \& Beltzer, 2002). Por otro lado, se ha reportado una disminución en los números de algunos rálidos como resultado de la pérdida y deterioro de su hábitat (Ripley \& Beehler, 1985; Eddleman et al., 1988; Taylor \& Van Perlo, 1988). Lo anterior resalta la importancia de localizar sus poblaciones como primer paso, para efectuar acciones encaminadas a su conservación.

El rascón pinto, Pardirallus maculatus (Boddaert, 1783), es una especie poco común en México, se encuentra desde Nayarit y Veracruz hasta Chiapas y la Península de Yucatán, y está ausente en la mayor parte del centro del país, aunque se le puede encontrar en el Estado de México, Morelos y Puebla (Howell \& Webb, 1995). El hábitat de la especie en México lo constituyen humedales de agua dulce con vegetación, particularmente tulares (Typha); también campos inundados y canales de riego (Dickerman \& Parkes, 1969; Dickerman, 1971; Scott et al., 1985; Howell \& Webb, 1995; González Bernal et al., 2006).

En Veracruz, se distribuye principalmente cercano a la costa del Golfo (Dickerman \& Parkes, 1969; Howell \& Webb, 1995; Schaldach, 1998-2003; eBird, 2019). En este trabajo se presentan registros de la especie efectuados en el centro-occidente de Veracruz, en áreas fuera de la distribución antes mencionada y observaciones sobre su alimentación, la cual ha sido poco documentada en México.

Se registró en tres fechas durante abril y mayo de 2014 un individuo (presumiblemente el mismo, con base en las características observadas) de P. maculatus en Peñuela, Amatlán de los Reyes (18 $51^{\circ}$ ' 43" N; $96^{\circ}$ 54' 25" O; 770 msnm; Fig. 1). Se observó al ave en un estanque perenne de aproximadamente 100 $\mathrm{m}^{2}$ en cuya orilla había pastos y tules (Typha domingensis Pers.) y sobre su superficie, abundantes lentejas de agua (familia Lemnaceae); dicho cuerpo de agua es alimentado por escurrimientos durante la época de lluvias. El paisaje en que se ubica el estanque está conformado en su mayor parte por cañaverales (Saccharum officinarum L.) con algunas zonas arboladas, principalmente a lo largo de arroyos y como cercos vivos; los alrededores del sitio están escasamente poblados, sin embargo, se encuentra relativamente cerca a centros urbanos, como Peñuela $(1 \mathrm{~km})$ y Buena Vista $(0.5 \mathrm{~km})$.

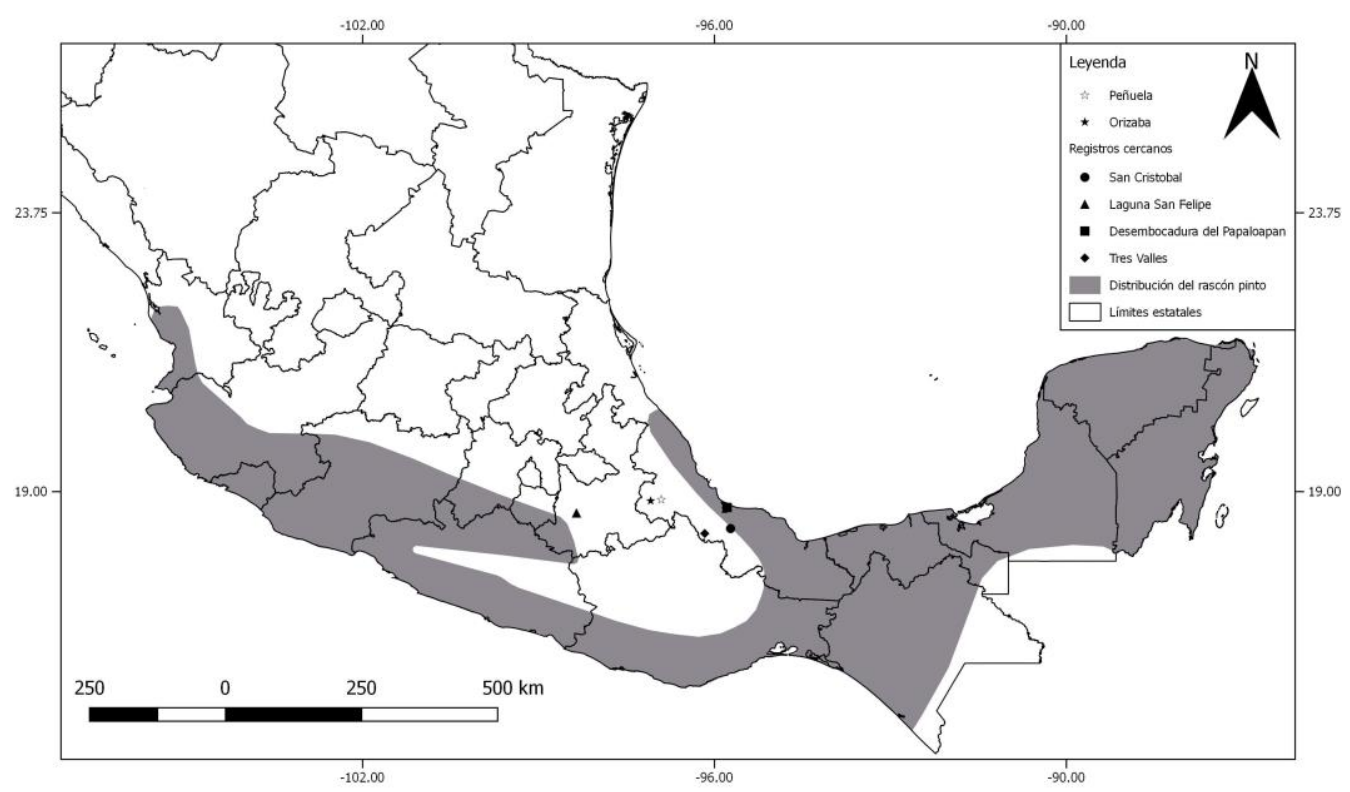

Figura 1. Nuevos registros del rascón pinto (Pardirallus maculatus) en el centro-occidente de Veracruz. Se indica el área de distribución de la especie (IUCN, 2019) así como los registros más cercanos a los presentados (Dickerman \& Parkes, 1969; Dickerman, 1971; Schaldach 1998-2003; IBUNAM 2007; ebird, 2019). 
El 29 de abril a las 9:50 a.m. se observó al rascón perchado sobre un tronco parcialmente sumergido en el borde del estanque, el ave permaneció ahí por algunos minutos (Fig. 2a) y posteriormente se ocultó entre la vegetación. El 7 de mayo a las 11:30 a.m. se le observó alimentarse: el rálido picoteaba las lentejas de agua mientras vadeaba por la orilla del estanque, en este lugar el agua solo alcanzaba a cubrirle hasta la parte baja del tarso (Fig. 2b). El 20 de mayo a las 10:40 a.m., se le observó desplazarse entre la vegetación, aparentemente, en busca de alimento. No se le encontró en visitas posteriores al estanque, el cual fue recorrido dos veces el mes siguiente y de forma irregular los siguientes dos años.

El segundo registro tuvo lugar el 16 de febrero de 2015, a unos $20 \mathrm{~km}$ al oeste del sitio en Peñuela. Se nos informó de un $P$. maculatus que llegó al patio de un domicilio particular en las afueras de la ciudad de Orizaba ( $18^{\circ} 50^{\prime} 28^{\prime \prime} \mathrm{N}$; $97^{\circ} 5^{\prime} 16^{\prime \prime} \mathrm{O}$; $1,230 \mathrm{msnm}$; Fig. 1). Cabe mencionar que, aunque este registro ocurrió en una zona urbana, Orizaba cuenta con numerosos cuerpos y corrientes de agua de donde podría haber provenido este organismo. El ave fue capturada y dado que se encontraba en buena condición física, se le liberó en un cuerpo de agua rodeado por pastos altos, la laguna el Novillero ( $18^{\circ} 53^{\prime} 3^{\prime \prime}$ N; $97^{\circ} 6^{\prime} 3^{\prime \prime} \mathrm{O}$; 1,300 msnm; Figura 2c), a unos $5 \mathrm{~km}$ de distancia.

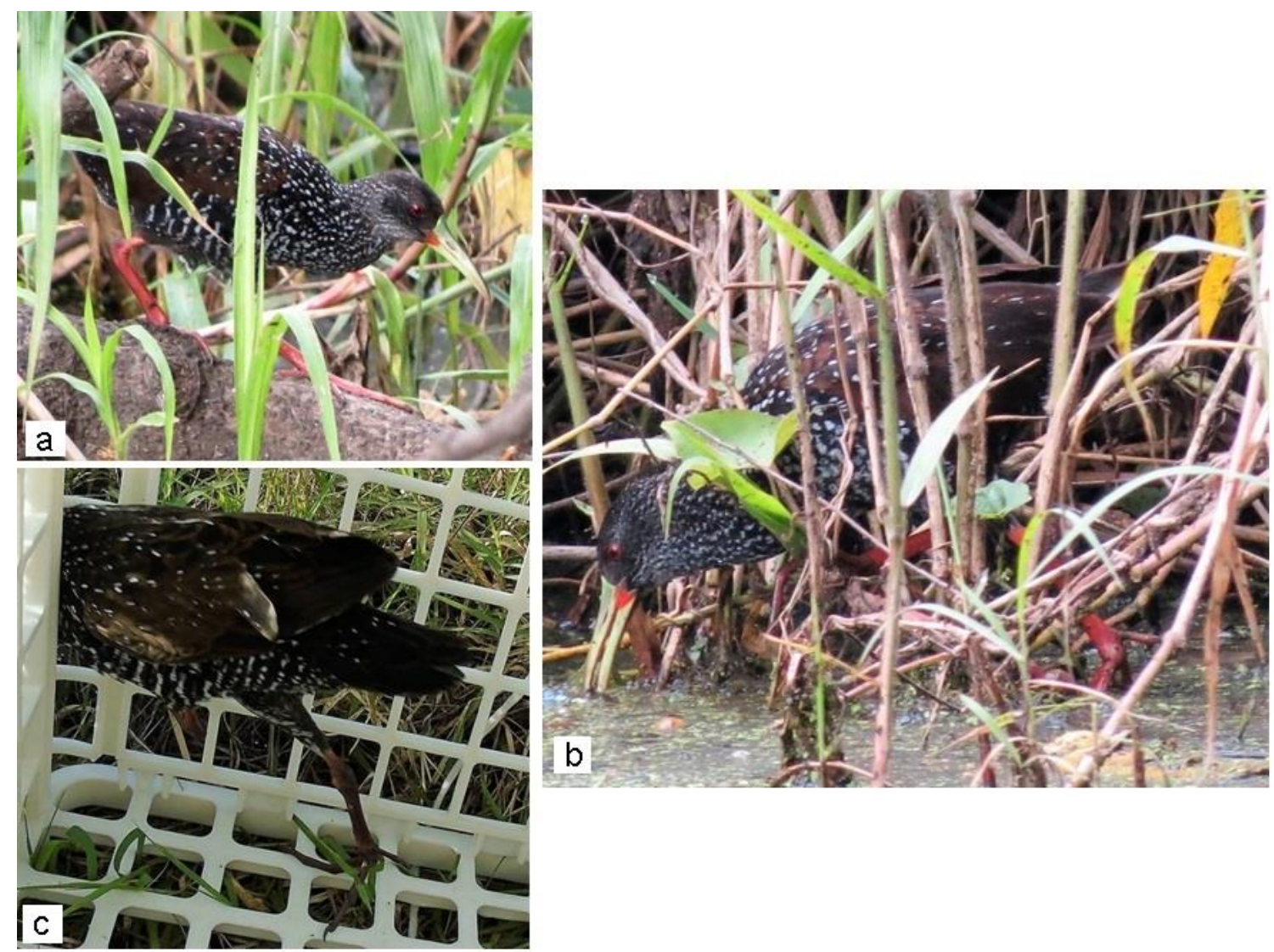

Figura 2. a) Rascón pinto observado en un estanque en Peñuela, Amatlán de los Reyes. b) Rascón pinto alimentándose de lentejas de agua en Peñuela. c) Liberación del rascón pinto encontrado en Orizaba.

Los dos rascones observados tenían el plumaje negruzco y densamente manchado de blanco (Fig. 2), como se ha descrito para los adultos de la especie (Howell \& Webb, 1995), el cual es distinto del plumaje café oscuro con solo algunas manchas blancas propio de los jóvenes de la subespecie que habita en México (P. m. insolitus [Bangs \& Peck, 1908]) (Dickerman \& Parkes, 1969; Howell \& Webb, 1995). Sin embargo, 
el color de los ojos y de las patas también puede ayudar a identificar la edad en esta familia (Pyle, 2008). Así, el rascón de Peñuela tenía los ojos rojos y las patas color rojo rosáceo brillante, por lo que corresponde en su totalidad con la descripción del adulto (Howell \& Webb, 1995), mientras que el ave de Orizaba, tenía las patas rosa-grisáceas, por lo que se trataría de un inmaduro.

Al considerar también las características del plumaje, y de acuerdo con la clasificación de edades de Pyle (2008), el rascón de Peñuela podría encontrarse entre las categorías AHY (After Hatching Year = Después del Año de Eclosión) y ASY (After Second Year = Después del Segundo Año), ya que las plumas del ala son uniformemente básicas, con rémiges relativamente brillantes y nuevas, además de contar con el color de ojos característico de los adultos que especies emparentadas adquieren hasta después del primer año; por otro lado, el ave de Orizaba se encontraría entre HY (Hatching Year = Año del Eclosión) y SY (Second Year = Segundo Año) ya que muestra cobertoras y plumas de vuelo desgastadas (plumaje de inmaduro) contrastante con las plumas del cuerpo ya remplazadas por plumas nuevas (Pyle, 2008).

Las observaciones presentadas constituyen los primeros registros de la especie en el centrooccidente del estado de Veracruz y se sitúan a 70 km (Peñuela) y $90 \mathrm{~km}$ (Orizaba) del límite de distribución señalado anteriormente (Howell \& Webb, 1995; IUCN, 2019), a 90 y $110 \mathrm{~km}$, respectivamente, de los registros más cercanos en la vertiente del Golfo de México (Dickerman \& Parkes, 1969; Schaldach, 19982003; IBUNAM, 2007), y a 130 y $150 \mathrm{~km}$, respectivamente, de la localidad más cercana en el centro de México (Dickerman, 1971; ebird, 2019).

La gran capacidad de vagabundeo y dispersión de los rálidos les permite llegar a sitios con hábitat apropiado atravesando grandes distancias, incluso han colonizado islas oceánicas (Ripley \& Beehler, 1985; García-R. \& Trewick, 2015). Pardirallus maculatus, es un vagabundo notable, se le ha registrado a más de $2,700 \mathrm{~km}$ del límite de su distribución, tanto al norte como al sur y en mar abierto a más de $300 \mathrm{~km}$ de la costa (Parkes et al., 1978), así pues, es una posibilidad que las aves observadas fueran individuos vagabundos. Por otro lado, también es posible que este rascón tenga una distribución más amplia que la conocida y que a causa de su conducta elusiva exista un sesgo en los registros de la especie (Parkes et al., 1978; Howell \& Webb, 1995); debido a que en el área en que se obtuvieron los registros existen diversos cuerpos y corrientes de agua que podrían brindar hábitat adecuado a esta especie, parece una explicación factible.

Pardirallus maculatus se alimenta de caracoles, insectos, lombrices, peces, anfibios (Birkenholz \& Jenni, 1964; Scott et al., 1985; Del Barco \& Beltzer, 2002; Lucero, 2012) y solo una planta se ha reportado como parte de su dieta, la herbácea acuática Potamogeton epihydrus Raf. (Parkes et al., 1978). Sin embargo, las lemnáceas han sido indicadas como alimento de otros miembros de Rallidae (Ripley \& Beehler, 1985; Taylor \& Van Perlo, 1988). Respecto a la estrategia de alimentación observada, aunque el picoteo no había sido registrado para $P$. maculatus, se sabe que es utilizado por varios rálidos para ingerir plantas flotantes e invertebrados que se encuentran sobre ellas (Beltzer \& Quiroga, 2007).

Los nuevos registros pueden ayudar a entender la biología evolutiva de las especies, identificar cambios en sus áreas de distribución y contribuir a la conservación, al reportar poblaciones aisladas o poco abundantes que puedan requerir protección (Monroy-Ojeda \& Isern, 2013; Sánchez-González, 2013). Dado que las localidades de $P$. maculatus en México son relativamente escasas y su tendencia poblacional es indeterminada (IUCN, 2019), los registros aquí presentados son relevantes, ya que pueden dar pie a estudios posteriores sobre la abundancia de la especie en esta región de México. La información que se aporta sobre su hábitat y alimentación puede ser útil en estrategias de manejo de este rascón escasamente conocido.

Agradecimientos. Agradecemos a los revisores de este trabajo sus comentarios y sugerencias que ayudaron a mejorar este manuscrito. 


\section{LITERATURA CITADA}

Beltzer, A. H., Quiroga, M. A. (2007) Birds, pp. 363-378. In: Iriondo, M. H., Paggi, J. C., Parma, M. J. (Eds.). The Middle Paraná River: Limnology of a Subtropical Wetland. Springer. Alemania.

Birkenholz, D. E., Jenni, D. A. (1964) Observations on the spotted rail and pinnated bittern in Costa Rica. The Auk: Ornithological Advances, 81, 558-559. https://doi.org/10.2307/4082744

Del Barco, H. D., Beltzer, A. H. (2002) Dieta de Pardirallus maculatus (Aves: Rallidae) en el Paraná Medio (Santa Fe, Argentina). Revista FAVE-Ciencias Veterinarias, 1, 51-58. https://doi.org/10.14409/favecv.v1i2

Dickerman, R. W., Parkes, K. C. (1969) Juvenal plumage of the Spotted Rail (Rallus maculatus). The Wilson Bulletin, 81, 207-209.

Dickerman, R. W. (1971) Notes on various rails in Mexico. The Wilson Bulletin, 83, 49-56.

eBird (2019) eBird: Una base de datos en línea para la abundancia y distribución de las aves. eBird, Ithaca, New York. Disponible en: http://ebird.org. (fecha de acceso: 3 de marzo de 2019).

Eddleman, W. R., Knopf, F. L., Meanley, B., Reid, F. A., Zembal, R. (1988) Conservation of North American Rallids. The Willson Bulletin, 100, 458-475.

García-R., J. C., Trewick, S. A. (2015) Dispersal and speciation in purple swamphens (Rallidae: Porphyrio). The Auk: Ornithological Advances, 132 (1), 140-155. https://doi.org/10.1642/AUK-14-114.1

Gonzáles-Bernal, M. A., Castillo-Guerrero, J. A., Hernández-Celis, C. R., Vega, X. (2006) Primer registro del Rascón Pinto (Pardirallus maculatus) en Sinaloa, México. Huitzil, 7, 35-36.

Howell, S. N. G., Webb, S. (1995) A Guide to the Birds of Mexico and Northern Central America. Oxford University Press. Nueva York, EUA.

IBUNAM (2007) Pardirallus maculatus - IBUNAM:CNAV:AV024659. Instituto de Biología, Universidad Nacional Autónoma de México. en: http://unibio.unam.mx/collections/specimens/urn/IBUNAM:CNAV:AV024659. (Fecha de acceso: 8 de febrero de 2017).

IUCN (2019) The IUCN Red List of Threatened Species. Version 2018-2. Disponible en: www.iucnredlist.org. (Fecha de acceso 10 de marzo de 2019).

Lucero, F. (2012) Primer registro de Gallineta Overa (Pardirallus maculatus) para la provincia de San Juan y menciones sobres su aspecto alimentario. Ecoregistros, 2, 1-3.

Monroy-Ojeda, A., Isern, S. G. (2013) Noteworthy bird records in Sontecomapan, Veracruz, Mexico. Acta Zoológica Mexicana (nueva serie), 29, 666-676. https://doi.org/10.21829/azm.2013.2931605

Parkes, C. K., Kibee, D. P., Roth, L. E. (1978) First records of the Spotted Rail (Pardirallus maculatus) for the United States, Chile, Bolivia and Western México. American Birds, 32, 295-299.

Pyle, P. (2008) Identification Guide to North American Birds. Part II: Anatidae to Alcidae. Slate Creek Press. Point Reyes Station, California, EUA.

Ripley, S. D., Beehler, B. M. (1985) Rails of the World: A compilation of new information, 1975-1983. Smithsonian Contributions to Zoology, 417, 1-28.

Sánchez-González, L. A. (2013) Cuando un "nuevo registro" es realmente un nuevo registro: consideraciones para su publicación. Huitzil, 14, 17-21.

Schaldach, Jr. W. J. (1998-2003) A partially annotated and taxonomic checklist of the birds of the state of Veracruz, Mexico. Disponible en: www.catemaco.info/docs/schaldach/annotated.html. (Fecha de acceso: 6 de febrero de 2017).

Scott, P. E., Andrews, D. D., MacKinnon, B. (1985) Spotted Rail: first record from the Yucatan Peninsula, Mexico. American Birds, 39, 854.

Taylor, B., Van Perlo, B. (1988) Rails: A guide to rails, crakes, gallinules and coots of the world. Pica Press. Reino Unido. 